\title{
Explicit Cayley covers of Kautz digraphs
}

\author{
Josep M. Brunat* \\ Departament de Matemàtica Aplicada II \\ Universitat Politècnica de Catalunya \\ Josep.M.Brunat@upc.edu
}

Submitted: Mar 3, 2010; Accepted: Apr 27, 2011; Published: May 8, 2011

Mathematics Subject Classifications: 05E18, 05C25, 05C20

\begin{abstract}
Given a finite set $V$ and a set $S$ of permutations of $V$, the group action graph $\operatorname{GAG}(V, S)$ is the digraph with vertex set $V$ and $\operatorname{arcs}\left(v, v^{\sigma}\right)$ for all $v \in V$ and $\sigma \in S$. Let $\langle S\rangle$ be the group generated by $S$. The Cayley digraph $\operatorname{Cay}(\langle S\rangle, S)$ is called a Cayley cover of $\operatorname{GAG}(V, S)$. We define the Kautz digraphs as group action graphs and give an explicit construction of the corresponding Cayley cover. This is an answer to a problem posed by Heydemann in 1996.
\end{abstract}

\section{Introduction}

The importance of graph symmetry from theoretical and applied points of view has been emphasized many times; see, for instance, $[1,2,11,12,14]$. Furthermore, the idea of associating a Cayley digraph to a non-symmetric digraph in such a way that the properties of one gives information about the other has been frequently used. For instance, Fiol et al. $[7,8,9]$ have shown that, in the context of dynamic memory networks, the idea of associating a Cayley digraph on a permutation group on the set of vertices of the network plays a central role in a unified approach to the topic. The idea of symmetrization of a digraph is used by Espona and Serra in [6] to construct Cayley covers of the de Bruijn digraphs, and by Mansilla and Serra in the context of $k$-arc transitivity [15, 16]. The group action graphs defined by Annexstein et al. in [2], give a way to associate to each non-symmetric digraph a number of Cayley graphs.

The de Bruijn and Kautz digraphs are the iterated line digraphs from the complete digraph with and without loops, respectively [10]. They are dense digraphs, and they have high connectivity, and many other good properties [3]. But, in general, they are not symmetric. Serra and Fiol have calculated the permutation groups of the de Bruijn

\footnotetext{
${ }^{*}$ Work partially supported by Project Gen. Cat. DGR 2009SGR1040 and by Ministerio de Ciencia y Tecnología under project MICINT 400826.
} 
digraph [18]. From them, an explicit construction of the Cayley digraph associated to the de Bruijn digraphs as group action graphs is known. For Kautz digraphs $K(d, n)$, it is known for which values of $d$ and $n$ they are Cayley digraphs (see [4]), and, in the case that $d+1$ is a prime number, Mansilla and Serra [15] gave an explicit construction of the corresponding Cayley digraph.

The problem 47 posed by Heydemann in [13], consists in giving an explicit construction of the Cayley digraph associated to the Kautz digraphs $K(d, n)$ considered as group action graph. Our goal here is to solve this problem for all values of $d$ and $n$.

The paper is organized as follows. In the next section we give definitions, known results and a suitable representation of the Kautz digraphs $K(d, n)$. Section 3 is devoted to an explicit construction of a group $\Sigma=\Sigma(d, n)$ and a generating system $\mathcal{G}$ for $\Sigma$. In Section 4 we show that the Cayley digraph Cay $(\Sigma, \mathcal{G})$ is a Cayley regular cover of $K(d, n)$, the explicit construction asked by Heydemann.

For undefined concepts about group theory we refer to [17], and for undefined concepts about graph theory we refer to [5].

\section{Group action graphs and Kautz digraphs}

Given a (finite) set $V$ and a set $S$ of permutations of $V$, the group action graph (GAG for short) defined by $V$ and $S$ is the digraph with vertex set $V$ and $\operatorname{arcs}\left(v, v^{\sigma}\right)$ for all $v \in V$ and $\sigma \in S$; it is denoted by $\operatorname{GAG}(V, S)$. If we admit multiple arcs, a GAG is regular, the degree being the cardinality of $S$. In a natural way, the group $\langle S\rangle$ generated by $S$ acts on $V$, and $\operatorname{GAG}(V, S)$ is strongly connected if and only if the action of $\langle S\rangle$ on $V$ is transitive. In this paper, all digraphs considered are strongly connected.

The concepts of arc-coloring and decompositions into permutations, are closely related to GAG. Let $\Gamma=(V, E)$ be a $d$-regular digraph. An arc-coloring of $\Gamma$ is an assignment of an element in $\{0, \ldots, d-1\}$ to each arc of $E$ in such a way that, for all $v \in V$, the $d$ arcs incident to $v$ have different assignments, and the $d$ arcs incident from $v$ have different assignments as well. The element assigned to an arc is called the color of the arc. A set $S=\left\{\sigma_{0}, \ldots, \sigma_{d-1}\right\}$ of $d$ permutations of $V$ is a decomposition into permutations of $\Gamma$ if, for every vertex $v \in V$, (i) $\left(v, v^{\sigma_{a}}\right) \in E$ for all $a \in\{0, \ldots, d-1\}$; (ii) $v^{\sigma_{a}}=v^{\sigma_{b}}$ implies $a=b$ for all $a, b \in\{0, \ldots, d-1\}$.

The two concepts, arc-coloring and decomposition into permutations, are equivalent. Indeed, given an arc-coloring of $\Gamma$, for each color $a \in\{0, \ldots, d-1\}$ and each $v \in V$, let $v^{\sigma_{a}}$ be the unique vertex adjacent from $v$ by an arc of color $a$. Then, the map $\sigma_{a}: V \rightarrow V$ defined by $v \mapsto v^{\sigma_{a}}$ is a permutation of $V$, and the set $S=\left\{\sigma_{0}, \ldots, \sigma_{d-1}\right\}$ is a decomposition of $\Gamma$ into permutations. Conversely, given a decomposition into permutations $S=\left\{\sigma_{0}, \ldots, \sigma_{d-1}\right\}$ of $\Gamma$, if we assign to the arc $\left(v, v^{\sigma_{a}}\right)$ the color $a$, then we obtain an arc-coloring of $\Gamma$. It is well known that, as a consequence of Hall's matching theorem, every $d$-regular digraph admits an arc-coloring (in fact, in general, it admits many arc-colorings).

A decomposition into permutations $S$ of a $d$-regular digraph $\Gamma=(V, E)$ allows us to see $\Gamma$ as a GAG. Indeed, $\Gamma$ is just the GAG defined by $V$ and $S$, that is, $\Gamma=\operatorname{GAG}(V, S)$. 
As, in general, $\Gamma$ admits many decompositions into permutations, the digraph $\Gamma$ can be seen as a GAG in many ways.

Given a group $\Omega$, and a generating system $S$ for $\Omega$, the Cayley digraph Cay $(\Omega, S)$ is the digraph which has $\Omega$ as set of vertices and each vertex $v$ is adjacent to the vertices $v s$, with $s \in S$. If $\Gamma$ is a $d$-regular digraph and $S$ is a decomposition into permutations of $\Gamma$, the Cayley graph $\operatorname{Cay}(\langle S\rangle, S)$ is called a Cayley regular cover of $\Gamma$. As pointed out in [2], the digraph $\Gamma$ is a quotient digraph of each of its regular covers. Indeed, fixed a vertex $v$, the map $f: \operatorname{Cay}(\langle S\rangle, S) \rightarrow \Gamma$ defined by $f(\sigma)=v^{\sigma}$ is a digraph homomorphism onto $\Gamma$, and, for all $u \in V$, the set $f^{-1}(u)$ has the cardinality of the stabilizer in $\langle S\rangle$ of $v$.

The de Bruijn digraph $B(d, n)$ is the digraph with vertex set $\mathbb{Z}_{d}^{n}$ and each vertex $z_{0} \ldots z_{n-1}$ is adjacent to the $d$ vertices $z_{1} \ldots z_{n}$ with $z_{n} \in \mathbb{Z}_{d}$. Clearly, $B(d, n)$ is $d$-regular. The digraph $B(d, 1)$ is the complete digraph with loops on $d$ vertices $K_{d}^{+}=\operatorname{Cay}\left(\mathbb{Z}_{d}, \mathbb{Z}_{d}\right)$. For $n \geq 2$, the digraphs $B(d, n)$ are iterated line digraphs $B(d, n)=L B(d, n-1)=$ $L^{n-1} B(d, 1)$, see [10]. For $a \in \mathbb{Z}_{d}$, the map $\sigma_{a}: \mathbb{Z}_{d}^{n} \rightarrow \mathbb{Z}_{d}^{n}$ defined by $\left(z_{0} \ldots z_{n-1}\right)^{\sigma_{a}}=$ $z_{1} \ldots z_{n-1}\left(z_{0}+a\right)$ is a permutation of $\mathbb{Z}_{d}^{n}$, and the set $S=\left\{\sigma_{0}, \ldots, \sigma_{d-1}\right\}$ is a decomposition of $B(d, n)$ into permutations. The Cayley regular cover Cay $(\langle S\rangle, S)$ associated to these permutations is known, see [15].

The Kautz digraph $K(d, n)$ is the $d$-regular digraph with vertex set $V=\left\{z_{0} \ldots z_{n-1} \in\right.$ $\mathbb{Z}_{d+1}^{n}: z_{i} \neq z_{i+1}$ for $\left.i=0, \ldots, n-2\right\}$, and each vertex $z_{0} \ldots z_{n-1}$ is adjacent to the $d$ vertices $z_{1} \ldots z_{n-1} z_{n}$ with $z_{n} \in \mathbb{Z}_{d+1} \backslash\left\{z_{n-1}\right\}$. The digraph $K(d, 1)$ is the complete digraph without loops on $d+1$ vertices $K_{d+1}=\operatorname{Cay}\left(\mathbb{Z}_{d+1}, \mathbb{Z}_{d+1} \backslash\{0\}\right)$. For $n \geq 2$, the digraphs $K(d, n)$ are iterated line digraphs $K(d, n)=L K(d, n-1)=L^{n-1} K(d, 1)$, see [10]. The trivial case $d=1$ gives $K(1, n) \simeq K(1,1) \simeq \operatorname{Cay}\left(\mathbb{Z}_{2},\{1\}\right)$, so in what follows we assume $d \geq 2$. When $d+1$ is a prime number, Fiol et al. [8] give a representation of $K(d, n)$ and a decomposition of $K(d, n)$ into permutations for which the corresponding Cayley regular cover is explicitly obtained by Mansilla and Serra in [15]. Our explicit construction for all values of $(d, n)$ is based in a similar description of the Kautz digraph and in a decomposition into permutations which uses this description.

To avoid inconsistencies of notation, for an integer $m \geq 2$, we take the set of integers $\{0,1, \ldots, m-1\}$ (and not equivalence classes of integers) as the elements of the cyclic group $\mathbb{Z}_{m}$ of order $m$ generated by 1 . In this way, as a set $\mathbb{Z}_{d}$ is a subset (but not a subgroup) of $\mathbb{Z}_{d+1}$ and, if $c \in \mathbb{Z}_{d}$ and and $z \in \mathbb{Z}_{d+1}$, the sum $z+c$ in $\mathbb{Z}_{d+1}$ has a non-ambigous meaning.

For $a \in \mathbb{Z}_{d}$, the map $\tau_{a}: \mathbb{Z}_{d+1} \rightarrow \mathbb{Z}_{d+1}$ defined by $x^{\tau_{a}}=x+a+1$ is a permutation of $\mathbb{Z}_{d+1}$, and the set $S=\left\{\tau_{0}, \ldots, \tau_{d-1}\right\}$ is a decomposition of $K_{d+1}$ into permutations, and the $\operatorname{arc}\left(z, z^{\tau_{a}}\right)$ is said to be of color $a$. As $K(d, n)=L^{n-1} K(d, 1)$, each vertex in $K(d, n)$ is a walk $z_{0} \ldots z_{n-1}$ in $K(d, 1)$, which is completely determined by the initial vertex $z_{0}$ and the sequence of colors $\mathbf{c}=c_{0} \ldots c_{n-2}$ of the successive $\operatorname{arcs}\left(z_{0}, z_{1}\right), \ldots,\left(z_{n-2}, z_{n-1}\right)$ in $K(d, 1)$. Denoting the vertex $z_{0} \ldots z_{n-1}$ of $K(d, n)$ by $\left(z_{0}, \mathbf{c}\right)=\left(z_{0}, c_{0} \ldots c_{n-2}\right)$, the vertex set of $K(d, n)$ can be identified with $\mathbb{Z}_{d+1} \times \mathbb{Z}_{d}^{n-1}$, and each vertex $\left(z, c_{0} \ldots c_{n-2}\right)$ is adjacent in $K(d, n)$ to the $d$ vertices $\left(z+c_{0}+1, c_{1} \ldots c_{n-2} c_{n-1}\right)$ with $c_{n-1} \in \mathbb{Z}_{d}$. From now on, we take this description for $K(d, n)$.

Let $V=\mathbb{Z}_{d+1} \times \mathbb{Z}_{d}^{n-1}$ be the vertex set of $K(d, n)$. For each $a \in \mathbb{Z}_{d}$, the map $\sigma_{a}: V \rightarrow V$ 
defined by $\left(z, c_{0} \ldots c_{n-2}\right)^{\sigma_{a}}=\left(x+c_{0}+1, c_{1} \ldots c_{n-2}\left(c_{0}+a\right)\right)$ is a permutation of $V$, and the set $S=\left\{\sigma_{0}, \ldots, \sigma_{d-1}\right\}$ is a decomposition of $K(d, n)$ into permutations. Thus, $K(d, n)$ is the group action graph $K(d, n)=\operatorname{GAG}(V, S)$. In what follows we give an explicit description of the Cayley regular cover Cay $(\langle S\rangle, S)$ of $K(d, n)=\operatorname{GAG}(V, S)$.

\section{The group}

Let $\psi$ be the shift automorphism of $\mathbb{Z}_{d}^{n-1}$ defined by $\psi\left(a_{0}, \ldots, a_{n-2}\right)=\left(a_{1}, \ldots, a_{n-2}, a_{0}\right)$. The map $i \mapsto \psi^{i}$ is a group homomorphism from $\mathbb{Z}_{n-1}$ to Aut $\mathbb{Z}_{d}^{n-1}$, the automorphism group of $\mathbb{Z}_{d}^{n-1}$, so we can form the semidirect product $\mathbb{Z}_{n-1} \rtimes \mathbb{Z}_{d}^{n-1}$ with the operation defined by $(i, \mathbf{a})(j, \mathbf{b})=\left(i+j, \psi^{j}(\mathbf{a})+\mathbf{b}\right)$.

Let $H_{d+1}^{d}$ be the subgroup of $\mathbb{Z}_{d+1}^{d}$ formed by the elements with sum of coordinates equal to zero:

$$
H_{d+1}^{d}=\left\{\left(x_{0}, \ldots, x_{d-1}\right) \in \mathbb{Z}_{d+1}^{d}: x_{0}+\cdots+x_{d-1}=0\right\} .
$$

The group $H_{d+1}^{d}$ has order $\left|H_{d+1}^{d}\right|=(d+1)^{d-1}$. Let $\phi$ be the shift automorphism of $H_{d+1}^{d}$ defined by $\phi\left(x_{0}, \ldots, x_{d-1}\right)=\left(x_{1}, \ldots, x_{d-1}, x_{0}\right)$. If $\mathbf{a}=\left(a_{0}, \ldots, a_{n-2}\right) \in \mathbb{Z}_{d}^{n-1}$, we define

$$
\phi^{\mathbf{a}}=\left(\phi^{a_{0}}, \phi^{a_{1}}, \ldots, \phi^{a_{n-2}}\right):\left(H_{d+1}^{d}\right)^{n-1} \rightarrow\left(H_{d+1}^{d}\right)^{n-1}
$$

by

$$
\phi^{\mathbf{a}}\left(\mathbf{x}_{0}, \ldots, \mathbf{x}_{n-2}\right)=\left(\phi^{a_{0}}\left(\mathbf{x}_{0}\right), \ldots, \phi^{a_{n-2}}\left(\mathbf{x}_{n-2}\right)\right) .
$$

Clearly, $\phi^{\mathbf{a}}$ is an automorphism of $\left(H_{d+1}^{d}\right)^{n-1}$. Denote by $\psi$ the shift automorphism of $\left(H_{d+1}^{d}\right)^{n-1}$ defined by $\psi\left(\mathbf{x}_{0}, \ldots, \mathbf{x}_{n-2}\right)=\left(\mathbf{x}_{1}, \ldots, \mathbf{x}_{n-2}, \mathbf{x}_{0}\right)$. (Note that we use the same symbol $\psi$ for the shift automorphism of $\mathbb{Z}_{d}^{n-1}$ and for the shift automorphism of $\left(H_{d+1}^{d}\right)^{n-1}$. In both cases $\psi$ is a shift of vectors of length $n-1$, while $\phi$ is applied to vectors of length d.) For each $(i, \mathbf{a}) \in \mathbb{Z}_{n-1} \rtimes \mathbb{Z}_{d}^{n-1}$, the map $\psi^{-i} \phi^{-\mathbf{a}}$ is an automorphism of $\left(H_{d+1}^{d}\right)^{n-1}$. Moreover, from the fact that $\phi^{\mathbf{a}} \psi=\psi \phi^{\psi^{-1}(\mathbf{a})}$, the map $f: \mathbb{Z}_{n-1} \rtimes\left(\mathbb{Z}_{d}\right)^{n-1} \rightarrow \operatorname{Aut}\left(H_{d+1}^{d}\right)^{n-1}$, defined by $f(i, \mathbf{a})=\psi^{-i} \phi^{-\mathbf{a}}$ is a group homomorphism. Indeed, we have

$$
\begin{aligned}
& f(i, \mathbf{a}) f(j, \mathbf{b})=\left(\psi^{-i} \phi^{-\mathbf{a}}\right)\left(\psi^{-j} \phi^{-\mathbf{b}}\right) \\
& =\psi^{-i}\left(\phi^{-\mathbf{a}} \psi^{-j}\right) \phi^{-\mathbf{b}} \\
& =\psi^{-i} \psi^{-j} \phi^{\psi^{j}(-\mathbf{a})} \phi^{-\mathbf{b}} \\
& =\psi^{-(i+j)} \phi^{-\left(\psi^{j}(\mathbf{a})+\mathbf{b}\right)} \\
& =f\left(i+j, \psi^{j}(\mathbf{a})+\mathbf{b}\right) \\
& =f((i, \mathbf{a})(j, \mathbf{b})) \text {. }
\end{aligned}
$$

Consider the semidirect product $\Sigma^{\prime}(d, n)=\left(\mathbb{Z}_{n-1} \rtimes \mathbb{Z}_{d}^{n-1}\right) \rtimes\left(H_{d+1}^{d}\right)^{n-1}$, with the operation defined by

$$
(i, \mathbf{a}, \mathbf{X})(j, \mathbf{b}, \mathbf{Y})=\left(i+j, \psi^{j}(\mathbf{a})+\mathbf{b}, \mathbf{X}+\psi^{-i} \phi^{-\mathbf{a}}(\mathbf{Y})\right)
$$


Let $\Sigma(d, n)$ be the direct product $\Sigma^{\prime}(d, n) \times \mathbb{Z}_{2}$ if $d$ is odd and $n$ is even, and $\Sigma(d, n)=$ $\Sigma^{\prime}(d, n)$ otherwise. The order of $\Sigma(d, n)$ is

$$
|\Sigma(d, n)|=\left\{\begin{array}{cl}
2(n-1) d^{n-1}(d+1)^{(d-1)(n-1)}, & \text { if } d \text { is odd and } n \text { is even; } \\
(n-1) d^{n-1}(d+1)^{(d-1)(n-1)}, & \text { otherwise. }
\end{array}\right.
$$

Now, we shall show a generating system for $\Sigma(d, n)$. First, let us introduce some notation to make it easier to write some elements in $\Sigma(d, n)$. Let $\mathbf{e}_{0}=10 \ldots 0, \ldots, \mathbf{e}_{n-2}=$ $0 \ldots 01$, be the vectors of the canonical base of $\mathbb{Z}_{d}^{n-1}$. If $a \in \mathbb{Z}_{d}$, then $a \mathbf{e}_{i}$ is the vector $0 \ldots 0 a 0 \ldots 0$ with $a$ in the $i$-th position (positions are counted from 0 to $n-2$ ). Both the zero vector of $\mathbb{Z}_{d}^{n-1}$ and the zero vector of $H_{d+1}^{d}$ are denoted by $\mathbf{0}$, while the zero vector of $\left(H_{d+1}^{d}\right)^{n-1}$ is denoted by $\mathbf{O}$. With this notation, the neutral element of $\Sigma(d, n)$ is $O=(0, \mathbf{0}, \mathbf{O}, 0)$ if $d$ is odd and $n$ even and $O=(0, \mathbf{0}, \mathbf{O})$ otherwise. We define $\mathbf{v}=$ $1 \ldots 12 \in H_{d+1}^{d}$ and $\mathbf{X}_{\mathbf{v}}=(\mathbf{v}, \mathbf{0}, \ldots, \mathbf{0}) \in\left(H_{d+1}^{d}\right)^{n-1}$. For each $j \in\{0, \ldots, d-2\}$, let $\mathbf{g}_{j}=0 \ldots 010 \ldots d$ be the vector of $H_{d+1}^{d}$ with the $j$-th coordinate equal to 1 , the last coordinate equal to $d=-1$, and the remaining coordinates equal to zero. Note that the vectors $\mathbf{g}_{0}, \ldots, \mathbf{g}_{d-2}$ form a generating system for $H_{d+1}^{d}$.

For each $a \in \mathbb{Z}_{d}$ define $G(a) \in \Sigma(d, n)$ by $G(a)=\left(1, a \mathbf{e}_{n-2}, \mathbf{X}_{\mathbf{v}}, 1\right)$ if $d$ is odd and $n$ is even, and $G(a)=\left(1, a \mathbf{e}_{n-2}, \mathbf{X}_{\mathbf{v}}\right)$ otherwise.

Proposition 1 The set $\{G(0), G(1)\}$ is a generating system for $\Sigma(d, n)$.

Proof It is sufficient to define elements $U, E(r)(0 \leq r \leq n-2)$, and $F(r, s)(0 \leq r \leq n-2$ and $0 \leq s \leq d-2)$, in the subgroup $\langle G(0), G(1)\rangle$ of $\Sigma=\Sigma(d, n)$ generated by $G(0)$ and $G(1)$ and to show that the elements $U, E(r)$ and $F(r, s)$ form a generating system for $\Sigma$.

First consider the case when $d$ is even or $n$ is odd, i.e., when $\Sigma$ does not have the factor $\mathbb{Z}_{2}$. Direct calculations give

$$
\begin{aligned}
G(1)^{n-2} & =(n-2,01 \ldots 1,(\mathbf{v}, \ldots, \mathbf{v}, \mathbf{0})) \\
G(0) G(1)^{n-2} & =(0,01 \ldots 1,(\mathbf{v}, \ldots, \mathbf{v})) \\
\left(G(0) G(1)^{n-2}\right)^{d} & =(0, \mathbf{0},(-\mathbf{v}, \mathbf{0}, \ldots, \mathbf{0}))=\left(0, \mathbf{0},-\mathbf{X}_{\mathbf{v}}\right) .
\end{aligned}
$$

Define $W=\left(G(0) G(1)^{n-2}\right)^{d}=\left(0, \mathbf{0},-\mathbf{X}_{\mathbf{v}}\right)$ and $U=W G(0)=(1, \mathbf{0}, \mathbf{O})$. Clearly, $U \in\langle G(0), G(1)\rangle$, and for any element $(i, \mathbf{a}, \mathbf{X}) \in \Sigma$, we have

$$
(i, \mathbf{a}, \mathbf{X}) U=(i+1, \mathbf{a}, \mathbf{X}) .
$$

Let $E(0)=W G(1) U^{-1}=\left(0, \mathbf{e}_{0}, \mathbf{O}\right)$ and, for $1 \leq r \leq n-2$, define

$$
E(r)=U^{r} E(0) U^{-r}=\left(0, \mathbf{e}_{r}, \mathbf{O}\right) .
$$

Then, $E(r) \in\langle G(0), G(1)\rangle$ and, for any element $(i, \mathbf{a}, \mathbf{X}) \in \Sigma$, we have

$$
(i, \mathbf{a}, \mathbf{X}) E(r)=\left(i, \mathbf{a}+\mathbf{e}_{r}, \mathbf{X}\right) .
$$


Now, for $0 \leq r \leq n-2$ and $0 \leq s \leq d-2$, we define

$$
\begin{aligned}
F(r, s) & =U^{r} W E(0)^{s+1} W^{-1} E(0)^{-(s+1)} \\
& =U^{r}\left(0,(s+1) \mathbf{e}_{0},-\mathbf{X}_{\mathbf{v}}\right)\left(0,-(s+1) \mathbf{e}_{0}, \mathbf{X}_{\mathbf{v}}\right) \\
& =(r, \mathbf{0}, \mathbf{O})\left(0, \mathbf{0},\left(\mathbf{g}_{s}, \mathbf{0}, \ldots, \mathbf{0}\right)\right) \\
& =\left(r, \mathbf{0}, \psi^{-r}\left(\mathbf{g}_{s}, \mathbf{0}, \ldots, \mathbf{0}\right)\right) .
\end{aligned}
$$

Clearly, $F(r, s) \in\langle G(0), G(1)\rangle$, and, for any element $(0, \mathbf{0}, \mathbf{X}) \in \Sigma$, we have

$$
(0, \mathbf{0}, \mathbf{X}) F(r, s)=\left(r, \mathbf{0}, \mathbf{X}+\psi^{-r}\left(\mathbf{g}_{s}, \mathbf{0}, \ldots, \mathbf{0}\right)\right) .
$$

Note that if $\mathbf{X}=\left(\mathbf{x}_{0}, \ldots, \mathbf{x}_{n-2}\right)$, then $\mathbf{X}+\psi^{-r}\left(\mathbf{g}_{s}, \mathbf{0}, \ldots, \mathbf{0}\right)$ is the vector obtained from $\mathbf{X}$ by adding $\mathbf{g}_{s}$ to $\mathbf{x}_{r}$, that is, by adding 1 to the $s$-th coordinate of $\mathbf{x}_{r}$ and -1 to the last coordinate of $\mathbf{x}_{r}$.

Let $(i, \mathbf{a}, \mathbf{X}) \in \Sigma$. Since $\mathbf{g}_{0}, \ldots, \mathbf{g}_{d-2}$ is a generating system for $H_{d+1}^{d}$, according to (3), an appropriate product of $F\left(r_{1}, s_{1}\right) \cdots F\left(r_{k}, s_{k}\right)$ gives an element of the form $(j, \mathbf{0}, \mathbf{X})$. Because of (2), multiplying on the right by an appropriate product $E\left(r_{1}\right) \cdots E\left(r_{\ell}\right)$ we can obtain $(j, \mathbf{a}, \mathbf{X})$. Finally, because of $(1)$, a product on the right by $U^{i-j}$ gives $(i, \mathbf{a}, \mathbf{X})$. We conclude that the elements $U, E(r)$, and $F(r, s)$ in $\langle G(0), G(1)\rangle$ form a generating system for $\Sigma$. Hence, $\langle G(0), G(1)\rangle=\Sigma$.

Now consider the case when $d$ is odd and $n$ is even; in this case, both $n-1$ and $d(n-1)$ are odd. Therefore,

$$
\begin{aligned}
G(1)^{n-1} & =(0,1 \ldots 1,(\mathbf{v}, \ldots, \mathbf{v}), 1), \\
G(1)^{(n-1) d} & =(0, \mathbf{0}, \mathbf{O}, 1) .
\end{aligned}
$$

Define $U, E(r)$ and $F(r, s)$ in terms of $G(0)$ and $G(1)$ in the same way as before. Given any element $A=(i, \mathbf{a}, \mathbf{X}, \alpha) \in \Sigma$, a suitable product of $F(r, s)$ 's, $E(r)$ 's and $U$ 's gives an element $(i, \mathbf{a}, \mathbf{X}, \beta)$. Now, multiplying by $G(1)^{(n-1) d}$ if necessary, we obtain $A$. So, in this case, $\Sigma=\langle G(0), G(1)\rangle$, as well.

By Proposition 1 , it is clear that the set $S=\{G(0), \ldots, G(d-1)\}$ is a generating system for $\Sigma(d, n)$, too.

\section{The Cayley cover}

Recall that we have defined $K(d, n)$ as the digraph with $V=\mathbb{Z}_{d+1} \times \mathbb{Z}_{d}^{n-1}$ as vertex set and each vertex $\left(z, c_{0} \ldots c_{n-2}\right)$ adjacent to the vertices $\left(z+c_{0}+1, c_{1} \ldots c_{n-2} c_{n-1}\right)$ with $c_{n-1} \in \mathbb{Z}_{d}$. Moreover, we consider the arc-coloring corresponding to the permutations $\sigma_{a}$ defined by $\left(z, c_{0} \ldots c_{n-2}\right)^{\sigma_{a}}=\left(z+c_{0}+1, c_{1} \ldots c_{n-2}\left(c_{0}+a\right)\right)$.

To define an action of the group $\Sigma=\Sigma(d, n)$ constructed in the previous section on $V$, the following notation will be used. Let $\mathbf{h}=(1, \ldots, d-2, d-1,0) \in \mathbb{Z}_{d+1}^{d}$. For $\mathbf{x}=\left(x_{0}, \ldots, x_{d-1}\right) \in H_{d+1}^{d}$, define $\mathbf{h} \cdot \mathbf{x}=x_{0}+2 x_{1}+\cdots+(d-1) x_{d-2}$ with operations in $\mathbb{Z}_{d+1}$. Moreover, when $d$ is odd, we denote by $m$ the element $m=(d+1) / 2$ of $\mathbb{Z}_{d+1}$.

The action is defined depending on the parity of $d$ and $n$. Define $\rho: V \times \Sigma \rightarrow V$, $\rho(v, A)=v A$ as follows: 
- if $d$ is even,

$$
(z, \mathbf{c})(i, \mathbf{a}, \mathbf{X})=\left(z+\mathbf{h} \cdot \sum_{\ell=0}^{n-2} \phi^{-c_{\ell}}\left(\mathbf{x}_{\ell}\right), \mathbf{a}+\psi^{i}(\mathbf{c})\right) ;
$$

- if $d$ and $n$ are odd,

$$
(z, \mathbf{c})(i, \mathbf{a}, \mathbf{X})=\left(z+\mathbf{h} \cdot \sum_{\ell=0}^{n-2} \phi^{-c_{\ell}}\left(\mathbf{x}_{\ell}\right)+\varepsilon(i) m, \mathbf{a}+\psi^{i}(\mathbf{c})\right),
$$

where $\varepsilon(i)=0$ if $i$ is even and $\varepsilon(i)=1$ if $i$ is odd.

- if $d$ is odd and $n$ is even,

$$
(z, \mathbf{c})(i, \mathbf{a}, \mathbf{X}, \alpha)=\left(z+\mathbf{h} \cdot \sum_{\ell=0}^{n-2} \phi^{-c_{\ell}}\left(\mathbf{x}_{\ell}\right)+\alpha m, \mathbf{a}+\psi^{i}(\mathbf{c})\right) .
$$

Then, we have:

Proposition 2 The map $\rho$ is a faithful action of $\Sigma$ on $V$.

Proof First, we show that $\rho$ is an action. In all cases it is easy to check that if $O$ is the neutral element in $\Sigma$, then $\rho(v, O)=v$ for every vertex $v$. We shall check that $\rho(\rho(v, A), B)=\rho(v, A B))$ for every vertex $v$ and all $A, B \in \Sigma$. Consider first the case when $d$ is even, and put $v=(z, \mathbf{c}), A=(i, \mathbf{a}, \mathbf{X})$ and $B=(j, \mathbf{b}, \mathbf{Y})$. We have,

$$
\begin{aligned}
{[(z, \mathbf{c})(i, \mathbf{a}, \mathbf{X})](j, \mathbf{b}, \mathbf{Y})=} & \left(z+\mathbf{h} \cdot \sum_{\ell=0}^{n-2} \phi^{-c_{\ell}}\left(\mathbf{x}_{\ell}\right), \mathbf{a}+\psi^{i}(\mathbf{c})\right)(j, \mathbf{b}, \mathbf{Y}) \\
= & \left(z+\mathbf{h} \cdot \sum_{\ell=0}^{n-2} \phi^{-c_{\ell}}\left(\mathbf{x}_{\ell}\right)+\mathbf{h} \cdot \sum_{\ell=0}^{n-2} \phi^{-a_{\ell}-c_{\ell+i}}\left(\mathbf{y}_{\ell}\right),\right. \\
& \left.\psi^{j}\left(\mathbf{a}+\psi^{i}(\mathbf{c})\right)+\mathbf{b}\right) . \\
= & \left(z+\mathbf{h} \cdot \sum_{\ell=0}^{n-2} \phi^{-c_{\ell}}\left(\mathbf{x}_{\ell}\right)+\mathbf{h} \cdot \sum_{\ell=0}^{n-2} \phi^{-a_{\ell-i}-c_{\ell}}\left(\mathbf{y}_{\ell-i}\right),\right. \\
& \left.\psi^{j}(\mathbf{a})+\psi^{i+j}(\mathbf{c})+\mathbf{b}\right) . \\
= & \left(z+\mathbf{h} \cdot \sum_{\ell=0}^{n-2} \phi^{-c_{\ell}}\left(\mathbf{x}_{\ell}+\phi^{-a_{\ell-i}}\left(\mathbf{y}_{\ell-i}\right)\right),\right. \\
& \left.\psi^{i+j}(\mathbf{c})+\psi^{j}(\mathbf{a})+\mathbf{b}\right) \\
= & (z, \mathbf{c})\left(i+j, \psi^{j}(\mathbf{a})+\mathbf{b}, \mathbf{X}+\psi^{-i} \phi^{-\mathbf{a}}(\mathbf{Y})\right) \\
= & (z, \mathbf{c})[(i, \mathbf{a}, \mathbf{X})(j, \mathbf{b}, \mathbf{Y})] .
\end{aligned}
$$


Consider the second case, when both $d$ and $n$ are odd. By the same argument as in the case $d$ even, now one must add $\varepsilon(i) m+\varepsilon(j) m$ to the initial vertex of $[(z, \mathbf{c}) A] B$. Since $m$ has order 2 , we have $m \varepsilon(i)+m \varepsilon(j)=m \varepsilon(i+j)$, which is what must be added to the initial vertex of $(z, \mathbf{c})(A B)$. Thus, in this case, $\rho$ is an action, as well.

An analogous argument applies to the third case, when $d$ is odd and $n$ is even. If $A=(i, \mathbf{a}, \mathbf{X}, \alpha)$ and $B=(j, \mathbf{B}, \mathbf{Y}, \beta)$, one must add $m \alpha+m \beta$ to the initial vertex of $[(z, \mathbf{c}) A] B$, and $m(\alpha+\beta)=m \alpha+m \beta$, to the initial vertex of $(z, \mathbf{c})(A B)$.

Now we shall see that the action is faithful. Consider first the case when $d$ is even. Assume that $(z, \mathbf{c})(i, \mathbf{a}, \mathbf{X})=(z, \mathbf{c})$ for all vertices $(z, \mathbf{c})$. By taking $\mathbf{c}=\mathbf{0}$, we get $\mathbf{0}=\mathbf{c}=\mathbf{a}+\psi^{i}(\mathbf{c})=\mathbf{a}+\mathbf{0}=\mathbf{a}$. For $\mathbf{c}=\mathbf{e}_{0}$, we have $\mathbf{e}_{0}=\mathbf{a}+\psi^{i}\left(\mathbf{e}_{0}\right)=\psi^{i}\left(\mathbf{e}_{\mathbf{0}}\right)$, so $i=0$. Finally, take $z=0$ and $\mathbf{c}=q \mathbf{e}_{j}$. Then

$$
0=\mathbf{h} \cdot \sum_{\ell=0}^{n-2} \phi^{-c_{\ell}}\left(\mathbf{x}_{\ell}\right)=\mathbf{h} \cdot\left(\phi^{-q}\left(\mathbf{x}_{\ell}\right)+\sum_{\ell \neq j} \mathbf{x}_{\ell}\right) .
$$

Analogously, for $z=0$ and $\mathbf{c}=(q+1) \mathbf{e}_{j}$, we have

$$
0=\mathbf{h} \cdot \sum_{\ell=0}^{n-2} \phi^{-c_{\ell}}\left(\mathbf{x}_{\ell}\right)=\mathbf{h} \cdot\left(\phi^{-q-1}\left(\mathbf{x}_{\ell}\right)+\sum_{\ell \neq j} \mathbf{x}_{\ell}\right) .
$$

Subtracting (5) from (4), we obtain

$$
\mathbf{h} \cdot\left(\phi^{-q}\left(\mathbf{x}_{j}\right)-\phi^{-q-1}\left(\mathbf{x}_{j}\right)\right)=0 .
$$

If $\mathbf{y}=\left(y_{0}, \ldots, y_{d-1}\right)=\phi^{-q}\left(\mathbf{x}_{j}\right)$, since $\mathbf{y} \in H_{d+1}^{d}$, we have $y_{d-1}=-\left(y_{0}+\cdots+y_{d-2}\right)$. Then,

$$
\begin{aligned}
0 & =\mathbf{h} \cdot\left(\mathbf{y}-\phi^{-1}(\mathbf{y})\right) \\
& =1\left(y_{0}-y_{d-1}\right)+2\left(y_{1}-y_{0}\right)+\cdots+(d-2)\left(y_{d-1}-y_{d-2}\right) \\
& =-y_{0}-y_{1}-\cdots-y_{d-2}-(d-1) y_{d-1} \\
& =-y_{d-1}-(d-1) y_{d-1} \\
& =y_{d-1} .
\end{aligned}
$$

Thus, the $(d-1)$-rst coordinate of $\mathbf{y}=\phi^{-q}\left(\mathbf{x}_{j}\right)$ is 0 , that is, the $(j-q)$-th coordinate of $\mathbf{x}_{j}$ is zero. Since this is for all $j \in\{0, \ldots, n-1\}$ and $q \in\{0, \ldots, d-1\}$, we obtain that $\mathbf{X}=\mathbf{O}$.

Consider now the case when $d$ is odd. By the same argument as in the $d$ even case, the values $i=0$ and $\mathbf{a}=\mathbf{0}$ are deduced. If $n$ is also odd, then $m \varepsilon(i)=m \varepsilon(0)=0$, and equalities (4) and (5) can be obtained. By the same argument we get $\mathbf{X}=\mathbf{O}$. If $n$ is even, equations (4) and (5) must be changed to

$$
0=\mathbf{h} \cdot\left(\phi^{-q}\left(\mathbf{x}_{\ell}\right)+\sum_{\ell \neq j} \mathbf{x}_{\ell}\right)+m \alpha, \quad \text { and } \quad 0=\mathbf{h} \cdot\left(\phi^{-q-1}\left(\mathbf{x}_{\ell}\right)+\sum_{\ell \neq j} \mathbf{x}_{\ell}\right)+m \alpha,
$$


and, by subtraction, the equality (6) is obtained. In the same way, we get $\mathbf{X}=\mathbf{O}$. Then, we have $(0, \mathbf{0})=(0, \mathbf{0})(i, \mathbf{a}, \mathbf{O}, \alpha)=(0, \mathbf{0})(0, \mathbf{0}, \mathbf{O}, \alpha)=(m \alpha, \mathbf{0})$. As $\alpha \in\{0,1\}$ and $m$ is of order 2, we obtain $\alpha=0$.

Next, we check that the permutations on $V$ defined by the elements $G(a)$ in $\Sigma$ act correctly.

Proposition 3 For each $a \in \mathbb{Z}_{d}$ and each vertex $(z, \mathbf{c})$ of $K(d, n)$, the vertex adjacent from $(z, \mathbf{c})$ by an arc of color $a$ is $(z, \mathbf{c})^{\sigma_{a}}=(z, \mathbf{c}) G(a)$.

Proof Let $\mathbf{c}=c_{0} \ldots c_{n-2} \in \mathbb{Z}_{d}^{n-1}$ and $\mathbf{X}_{\mathbf{v}}=\left(\mathbf{x}_{0}, \ldots, \mathbf{x}_{n-2}\right)=(\mathbf{v}, \mathbf{0}, \ldots, \mathbf{0})$. First, we calculate $\mathbf{h} \cdot \sum_{\ell=0}^{n-2} \phi^{-c_{\ell}}\left(\mathbf{x}_{\ell}\right)$.

For even $d$,

$$
\begin{aligned}
\mathbf{h} \cdot \sum_{\ell=0}^{n-2} \phi^{-c_{\ell}}\left(\mathbf{x}_{\ell}\right) & =\mathbf{h} \cdot \phi^{-c_{0}}(\mathbf{v}) \\
& =(1+2+\cdots+(d-1))+c_{0} \\
& =(d-1) \frac{d}{2}+c_{0} \\
& =(d+1) \frac{d-2}{2}+1+c_{0} \\
& =1+c_{0} .
\end{aligned}
$$

If $d$ and $n$ are odd,

$$
\begin{aligned}
\mathbf{h} \cdot \sum_{\ell=0}^{n-2} \phi^{-c_{\ell}}\left(\mathbf{x}_{\ell}\right)+\varepsilon(1) m & =(1+2+\cdots+(d-1))+c_{0}+m \\
& =d \frac{d-1}{2}+c_{0}+\frac{d+1}{2} \\
& =(d+1) \frac{d-1}{2}+1+c_{0} \\
& =1+c_{0} .
\end{aligned}
$$

Analogously, if $d$ is odd and $n$ is even,

$$
\mathbf{h} \cdot \sum_{\ell=0}^{n-2} \phi^{-c_{\ell}}\left(\mathbf{x}_{\ell}\right)+m=1+c_{0} .
$$

Thus, in any case,

$$
(z, \mathbf{c}) G(a)=\left(z+c_{0}+1, a \mathbf{e}_{n-2}+\phi(\mathbf{c})=\left(z+c_{0}+1, c_{1} \ldots c_{n-2}\left(c_{0}+a\right)\right)=(z, \mathbf{c})^{\sigma_{a}} .\right.
$$

Putting together the description of $K(d, n)$ as a GAG, and Propositions 1, 2, and 3, we have the main theorem: 
Theorem 1 (i) The Kautz digraph is the group action graph $K(d, n)=G A G(V, S)$ where $V=\mathbb{Z}_{d+1} \times \mathbb{Z}_{d}^{n-1}$, and $S$ is the set $S=\left\{\sigma_{a}: a \in \mathbb{Z}_{d}\right\}$ of permutations of $V$ defined by $\left(z, c_{0} \ldots c_{n-2}\right)^{\sigma_{a}}=\left(z+c_{0}+1, c_{1} \ldots c_{n-2}\left(c_{0}+a\right)\right)$.

(ii) The group generated by $S$ is $\Sigma(d, n)$, and

$$
\text { Cay }(\Sigma(d, n),\{G(0), \ldots, G(d-1)\})
$$

is a Cayley regular cover of $K(d, n)=\operatorname{GAG}(V, S)$.

\section{References}

[1] S. B. Akers and B. Krishnamurthy. A group theoretic model for symmetric interconnection networks. IEEE Trans. Comput., 38,4:555-565, 1989.

[2] F. Annexstein, M.Baumslag, and A.L. Rosenberg. Group action graphs and parallel architectures. SIAM J. Comput., 19:544-569, 1990.

[3] J. C. Bermond and C. Peyrat. De Bruijn and Kautz networks: A competitor for the hypercube? Hypercube Dist. Comput., 3:279-293, 1989.

[4] J. M. Brunat, M. Espona, M. A. Fiol, and O. Serra. On Cayley line digraphs. Discrete Math., 138:147-159, 1995.

[5] G. Chartrand and L. Lesniak. Graphs \& Digraphs. Chapman \& Hall, London, third edition, 1996.

[6] M. Espona and O. Serra. Cayley digraphs based on the de Bruijn networks. SIAM J. Discrete Math., 11(2):305-317, May 1998.

[7] M. A. Fiol, J. Fàbrega, O. Serra, and J. L. A. Yebra. A unified approach to the design and control of dynamic memory networks. Parallel Processing Letters, 3(4):445-456, 1993.

[8] M. A. Fiol, J. Fàbrega, and J. L. A. Yebra. Cayley and Kautz digraphs as models for dynamic memory networks. In M. H. Hamza, editor, Proceedings of the IASTED International Symposium: Applied Informatics, Innsbruck, 1986.

[9] M. A. Fiol, J. Fàbrega, and J. L. A. Yebra. The design and control of dynamic memory networks. In Proc. 1988 IEEE Int. Symp. on Circuits and Systems, pages 175-179, Espoo, Finland, June 1988.

[10] M. A. Fiol, J. L. A. Yebra, and I. Alegre. Line digraph iterations and the $(d, k)$ digraph problem. IEEE Trans. Comput., C-33:400-403, 1984.

[11] E. Fuller and B. Krishnamurthy. Symmetries in Graphs: An Annotated bibliography. Technical report, Computer Research Laboratory, Tektronics Laboratoires, Beaverton,OR, 1986.

[12] G. Hahn and G. Sabidussi, editors. Graph Symmetry: Algebraic Methods and Applications, volume 497 of NATO ASI Series C, Montréal, 1996. Kluwer Academic. 
[13] M. C. Heydemann. Cayley graphs and interconnection networks. In G. Hahn and G. Sabidussi, editors, Graph Symmetry: Algebraic Methods and Applications, volume 497 of NATO ASI Series C, pages 167-224, Montréal, 1996. Kluwer Academic.

[14] S. Lakshmivarahan, Jung Sing Jwo, and S. K. Dhall. Symmetry in interconnexion networks based on Cayley graphs of permutation groups: A survey. Parallel Computing, 19:361-407, 1993.

[15] S. P. Mansilla and O. Serra. Construction of $k$-arc transitive digraphs. Discrete Math., 231(1-3):337-349, 2001.

[16] S. P. Mansilla and O. Serra. Automorphism groups of $k$-arc transitive covers. Discrete Math., 276(1-3):273-285, 2004.

[17] D. J. S. Robinson. A Course in the Theory of Groups, volume 80 of Graduate Texts in Mathematics. Springer Verlag, New York, Heidelberg, second edition, 1982.

[18] O. Serra and M. A. Fiol. On some groups associated to arc-colored digraphs. In Combinatorics 88, volume 2 of Research and Lecture Notes in Mathematics, pages 413-420, Comenda di Rende, 1991. Mediterranean Press. 